\title{
A Potential of sFasL in Preventing Gland Injury in Sjogren's Syndrome
}

\author{
Jiao Luo, ${ }^{1,2}$ Ying Wang, ${ }^{3}$ Bing Yu, ${ }^{1,2}$ Hongyan Qian, ${ }^{1,2}$ Yan He, ${ }^{1,2}$ and Guixiu Shi ${ }^{1,2}$ \\ ${ }^{1}$ Department of Rheumatology and Clinical Immunology, The First Hospital of Xiamen University, Xiamen 361003, China \\ ${ }^{2}$ Xiamen Key Laboratory of Rheumatology and Clinical Immunology, Xiamen, China \\ ${ }^{3}$ The Chenggong Hospital Affiliated to Xiamen University, Xiamen, Fujian, China
}

Correspondence should be addressed to Yan He; swallowheyan@qq.com and Guixiu Shi; gshi@xmu.edu.cn

Received 26 October 2016; Accepted 6 February 2017; Published 23 February 2017

Academic Editor: Xiaoquan Rao

Copyright (C) 2017 Jiao Luo et al. This is an open access article distributed under the Creative Commons Attribution License, which permits unrestricted use, distribution, and reproduction in any medium, provided the original work is properly cited.

\begin{abstract}
Fas and its ligand FasL, members of tumor necrosis factor receptor superfamily, have been implicated in the process of cell apoptosis FasL consists of two forms, membrane FasL (mFasL) and soluble FasL (sFasL). sFasL can be produced by mFasL cleaved by matrix metalloproteinases (MMP) and also reveals a role for binding to Fas which is expressed on cell surface. Although Fas/FasL axis has been implicated in a variety of diseases, its role in Sjogren's syndrome still remains ill defined. In this study, we investigated the potential of sFasL in the pathogenesis of Sjogren's syndrome (SS). We found that the serum levels of sFasL in SS patients were significantly lower than healthy subjects. Moreover, serum levels of sFasL in patients with mild disease activity were higher than patients with severe disease activity. There is a positive correlation of the serum level of sFasL with uptake index of parotid gland in our expectation. In addition, liver injury involvement in SS patients showed decreased level of sFasL. Furthermore, we here also observed that the protective cytokine IL-10 expression was positively correlated with sFasL expression. Thus, our results here suggest a potential of sFasL in maintaining gland organ homeostasis.
\end{abstract}

\section{Introduction}

Sjogren's syndrome (SS) is a chronic autoimmune disease that resulted from immune tolerance breakdown, leading to lymphocytes infiltration in gland organs (salivary gland, lachrymal gland, and the liver) and immune complex deposition as a consequence of $\mathrm{B}$ cell hyperactivity [1-3]. The primary manifestation of SS is oral and ocular dryness characterized by lymphocytes infiltration of salivary and lachrymal glands in tissues leading to a progressive destruction of these glands. In addition, liver known as a large secretory organ was also often damaged by the abnormal immune response in SS patients. Autoimmune liver injury accounts for approximately $5 \%$, including primary biliary cirrhosis (PBC) and autoimmune hepatitis (AIH) [4-6].

Fas and its ligand (FasL) are members of tumor necrosis factor receptor superfamily [7]. FasL plays a critical role in the process of cell apoptosis. Human FasL is of 281 amino acids and consists of an 80-amino acid cytoplasmic domain, a 22amino acid transmembrane domain, and a large extracellular domain. The region of FasL exposed to the outside of the cells consists of 179 amino acids [8]. The extracellular domain is responsible for binding to its receptor Fas. FasL induces apoptotic death of sensitive lymphoid cells expressing its cell surface receptor [9]. Indeed, activated $\mathrm{T}$ and $\mathrm{B}$ lymphocytes express Fas receptor and thus are sensitive to Fas receptor mediated apoptosis $[9,10]$. This has been proposed to be responsible for several regulatory functions of the immune system, including tolerance acquisition, downregulation of immune reactions, and clonal deletion of peripheral lymphocytes [11-14]. Moreover, FasL can be catalyzed by matrix metalloproteinases (MMP) from membranes, which lead to a soluble form sFasL [15]. sFasL could also induce apoptosis of cells when it binds to Fas which is expressed on cell surface. Excessive expression of FasL can inhibit some autoimmune diseases by deleting autoreactive immune cells $[16,17]$. 
TABLE 1: Demographic data and clinical characteristics of subjects in the study.

\begin{tabular}{lcc}
\hline Characteristics & SS patients & Healthy control \\
\hline Total & 60 & 20 \\
Age at study mean (SD) years & 47 & 46 \\
Female sex & $57(95 \%)$ & $19(95 \%)$ \\
PBC (primary biliary cirrhosis) & $2(3.3 \%)$ & - \\
AIH (autoimmune hepatitis) & $4(6.7 \%)$ & - \\
ILD (interstitial lung disease) & $5(8.3 \%)$ & - \\
RTA (renal tubular acidosis) & $2(3.3 \%)$ & - \\
Hypothyroidism & $4(6.7 \%)$ & - \\
\hline
\end{tabular}

Previous findings reveal a role for sFasL in the development of diseases, while the detailed function in the pathogenesis of SS remains unknown clearly. In the present study, we found that serum levels of sFasL were significantly lower in SS patients with mild disease activity, and the levels of sFasL exhibited a positive correlation with uptake index of parotid gland. Furthermore, the SS patients with liver injury showed a decreased level of sFasL. These data suggested that sFasL might exhibit a preventive role in the gland injury in the pathogenesis of SS.

\section{Subjects and Methods}

2.1. Patients and Controls. A total of 60 patients diagnosed with SS (57 women and 3 men, age 22-69, mean 47 years) fulfilled the revised version of the European criteria for SS [18]. The patients were recruited from the outpatient clinic and ward of the Department of Rheumatology and Clinical Immunology, the First Hospital of Xiamen University. The results were compared with a population of 20 healthy volunteers (healthy controls) matched for sex and age. Local ethics committee approved the study and informed consent was obtained from patients and control subjects. The number and clinical characteristics of healthy controls and patients with SS were summarized in Table 1. The disease activity is performed by SSDAI score, assessed by constitutional symptoms, change in salivary gland swelling, articular symptoms, hematologic features, pleuropulmonary symptoms, change in vasculitis, active renal involvement, and peripheral neuropathy [19]. Total SSDAI score was 21. Often the disease activity was divided into active and stable state depending on the SSDIA score of 5 points $[19,20]$.

2.2. Detection of Serum Proteins. The protein production of sera from SS patients was determined by Luminex assay from eBioscience (San Diego, CA, USA).

2.3. Parotid Gland ECT. 43 patients who have Sjogren's syndrome have been injected with 99-mTcO4 through elbow vein and then dynamic salivary gland was scanned. They were orally given vitamin C $0.1 \mathrm{~g}$ at the fifteenth minute. Computer produced composite images including dynamic images, timeactivity curves, and functional index of all salivary glands were acquired automatically using the technique of region of interest (ROIs) and self-compiled software of the computer. Uptake and excretion index (\%) of parotid gland (PG) examined by $99 \mathrm{~m}$ Tcs was acquired.

2.4. Statistical Analysis. All data were analyzed in GraphPad Prism 5. Results are presented as mean \pm SEM. The Mann-Whitney $U$ test, Spearman's correlation analysis, and unpaired $t$-test with Welch's correction were used to calculate significance. Statistical significance was accepted for $P$ values $<0.05$.

\section{Results}

3.1. Clinical Characteristics of SS Patients. The clinical characteristics of SS patients were summarized for this study (Table 1). Sixty SS patients and twenty healthy control of Southern Chinese population were enrolled in this project. The mean age for SS patients was 47 years with range (23-69), including 57 females and 3 males. Among these 60 patients, there were 2 patients (3.3\%) with PBC, 4 patients $(6.7 \%)$ with AIH, 5 patients $(8.3 \%)$ with ILD, 2 patients $(3.3 \%)$ with RTA, and 4 patients $(6.7 \%)$ with hypothyroidism.

3.2. Decreased Serum sFasL Levels in SS Patients. To explore the role of sera cytokines in the pathogenesis of SS, the Luminex assay was conducted. Compared with healthy controls, significantly decreased serum sFasL levels in SS patients were observed $(P<0.0001)$. As shown in Figure 1 , the median level of sFasL in SS patients was $5.352 \mathrm{pg} / \mathrm{mL}$ with range of $0.47-30.63 \mathrm{pg} / \mathrm{mL}$, while in healthy controls it was $10.66 \mathrm{pg} / \mathrm{mL}(1.380-22.16 \mathrm{pg} / \mathrm{mL})$. Although it has been demonstrated that sFasL plays a critical role in many diseases, the role of sFasL in SS patients has not been clearly confirmed.

\subsection{Relation of Serum sFasL Levels with Disease Activity in SS} Patients. Regarding the alteration of sFasL in SS patients, the relation between disease activity and sFasL was investigated. The disease activity is performed by SSDAI score, which assessed a combination of clinical history, physical examination, organ specific functional tests, and serologic studies [21]. The SS patients were divided two groups depending on SSDAI score. Here, serum sFasL level in patients with mild disease activity $(6.849 \pm 1.120 \mathrm{pg} / \mathrm{mL})$ was higher than severe disease activity $(2.790 \pm 0.4326 \mathrm{pg} / \mathrm{mL})(P=0.043)$ (Figure 2). 
TABLE 2: Serum sFasL levels in liver damage.

\begin{tabular}{lccc}
\hline Group & Number $(n)$ & Serum sFasL $(\mathrm{pg} / \mathrm{mL})$ & $P$ value \\
\hline SS patients without PBC/AIH & 54 & $5.514 \pm 0.8254$ & - \\
SS patients with PBC & 2 & $5.005 \pm 0.8750$ & 0.7467 \\
SS patients with AIH & 4 & $2.940 \pm 0.7573$ & $0.038^{*}$ \\
\hline
\end{tabular}

${ }^{*} P$ values were obtained from the statistical comparisons of serum $s$ FasL levels among the various study groups. SS patients without PBC/AIH were compared with SS patients with PBC and with AIH. Unpaired $t$-test with Welch's correction was used. Statistical significance was accepted for $P$ values $<0.05$.

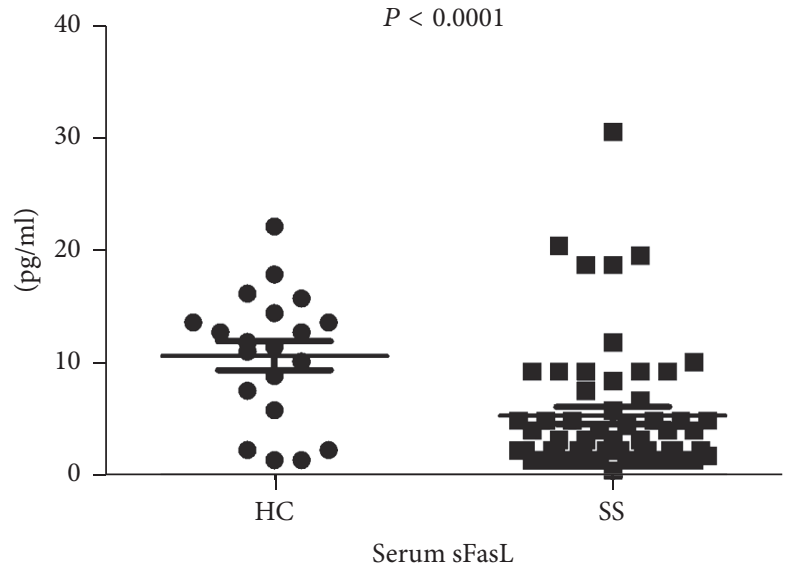

Figure 1: Reduced expression of sFasL in SS patient. The serum levels of sFasL in SS patients $(n=60)$ and healthy controls $(\mathrm{HC})$ $(n=20)$ were detected by Luminex assay. Each symbol represented an individual sample and horizontal lines showed median values. Mann-Whitney $U$ test was conducted to compare the data between two groups. $P<0.05$ was considered as statistic significance.

3.4. Correlation of Serum sFasL Levels with Uptake Index of Parotid Gland in SS Patients. 43 subjects of SS patients in this study have taken parotid gland ECT examinations for detecting the gland function. The role of sFasL in the SS patients with parotid gland was explored. As expected, in Figure 3, the sFasL levels were positively correlated with uptake index of parotid gland in the SS patients $(P=0.002$, $\left.r^{2}=0.2107\right)$.

3.5. Decreased Serum sFasL Expression in SS Patients with $A I H$. As a secretory organ, liver injury often occurs in SS patients. In our collective SS subjects, 2 SS patients were with $\mathrm{PBC}$ and $4 \mathrm{SS}$ patients were with AIH. Furthermore, the sFasL in these SS patients was detected. As expected, in keeping with the parotid gland, the SS patients without $\mathrm{PBC} / \mathrm{AIH}(5.514 \pm 0.8254)$ were higher than SS patients with AIH $(2.940 \pm 0.7573)(P=0.0338)$, although there was no significance in SS patients with PBC $(5.005 \pm 0.8750)(P=$ 0.7467) (Table 2).

3.6. Potential of sFasL in Modulating Regulatory Cytokine IL10 Expression. IL-10 is a critical protective cytokine in the development of SS [22]. Thus, the correlation of sFasL and IL-10 was analyzed. Interestingly, we demonstrated that there

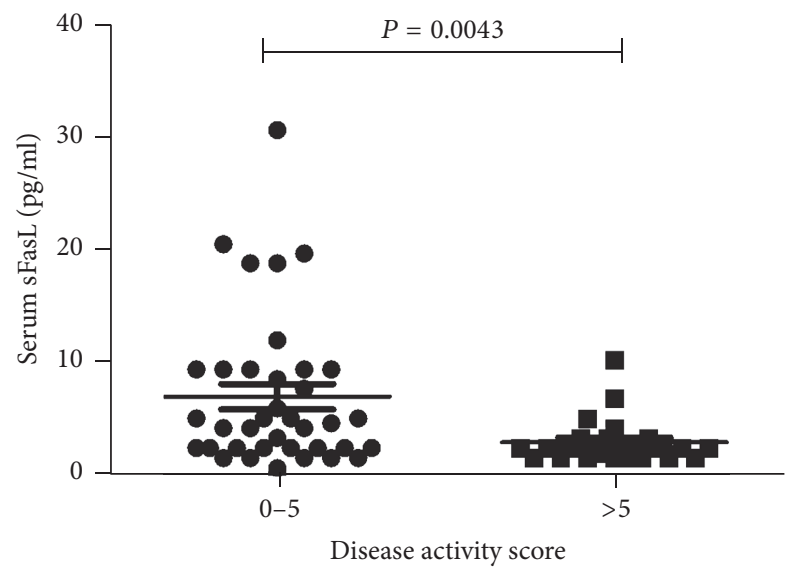

FIGURE 2: Decreased serum sFasL expression in patients with severe disease activity. The subjects in SS group were grouped into mild $(n=37)$ and severe $(n=23)$ disease activity dependent on the SSDAI score as described in methods. Total SSDAI score was performed when the serum was collected. Each symbol represented an individual sample and horizontal lines showed median values. Mann-Whitney $U$ test was conducted to compare the data respectively. $P$ value $<0.05$ was considered statistically significant.

is a positive correlation between sFasL and IL-10 $(P<0.0001$, $r^{2}=0.1530$ ) (Figure 4).

\section{Discussion}

In our study, we demonstrated the sFasL expression and its potential role in SS patients. Serum sFasL levels were significantly reduced in SS patients when compared with healthy control subjects. Furthermore, serum sFasL levels of patients in stable state were higher than patients in active state. In addition, we also found that the sFasL levels were positively correlated with uptake index of parotid gland in the SS patients and a decreased expression in SS patients with liver injury. Interestingly, a positive correlation between $\mathrm{sFasL}$ and IL-10 was investigated. These data suggested that sFasL might prevent damage of gland and plays a protective role in the pathogenesis of SS.

The natural course of the disease and the onset of salivary gland dysfunction were not clearly known [23]. Previous report has found that serum sFas and sFas-L levels were significantly higher in some rheumatic disease patients [24]. In children with chronic kidney disease (CKD), sFas/sFasL ratio may be a new marker of inflammation and endothelial dysfunction [25]. However, the role Fas/FasL axis in SS 


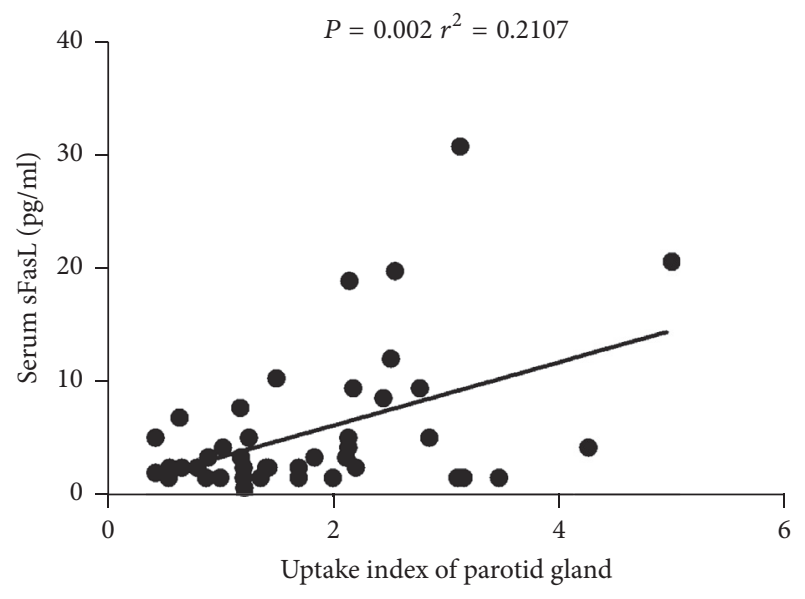

FIGURE 3: Positive correlation between sFasL and uptake index of parotid gland in SS patients. The determination of linear relationships between sFasL expression and uptake index of parotid gland in SS patients was performed by Spearman correlation coefficient. $P$ value $<0.05$ was considered statistically significant.

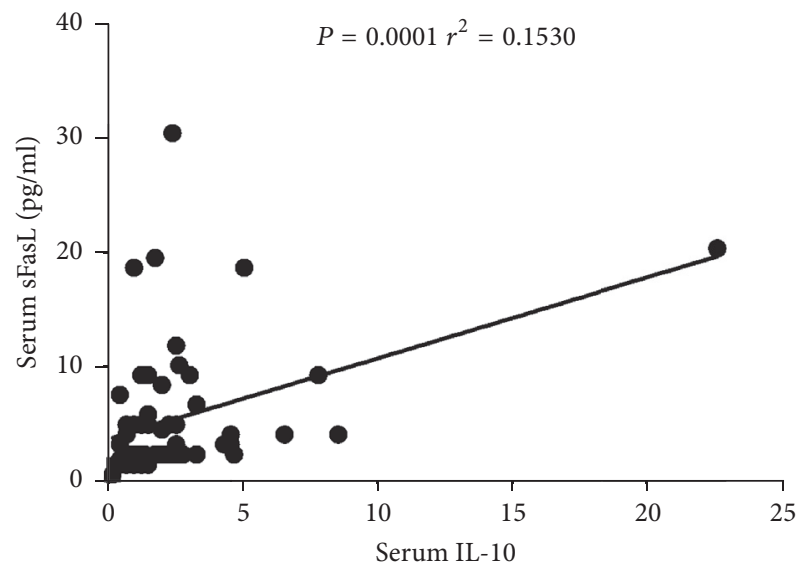

FIgURE 4: Positive correlation between sFasL with IL-10 in SS patients. The determination of linear relationships between sFasL expression and IL-10 in SS patients $(n=60)$ was performed by Spearman correlation coefficient.

which refers to keratoconjunctivitis sicca and xerostomia due to lymphocytic infiltrates of lachrymal and salivary glands remains unknown [26]. The glandular infiltration in SS is composed mainly of CD4+ T lymphocytes [27] and also contains a substantial number of B cells and plasma cells [28, 29]. The infiltration of lymphocytes into glandular aggregates apparently plays a crucial role in the tissue pathology of SS [30]. Apoptosis of activated T lymphocytes is essential in the regulation and timely resolution of inflammatory and immune responses [31]. It was evident that the interaction of Fas with FasL regulates a large number of pathophysiological processes of apoptosis including autoimmune diseases [32]. Accumulated evidences suggest an important role of apoptosis in the pathogenesis of SS [33]. Apoptosis of salivary glands $\mathrm{T}$ cells was decreased in SS patients [34]. It was demonstrated that sFasL treatment has potential therapeutic benefit in reducing inflammatory infiltrate and neovascularization in primary and recurrent forms of herpetic stromal keratitis and that it does so by augmenting the restriction of Fas+ inflammatory cells mediated by membrane FasL [35]. Previous study also provided evidence that sFasL could be related to the mechanism involved in the elimination of the parasite [36]. Some study also found that sFasL might mediate the apoptosis of T lymphocytes [37]. However, a study showed that levels of sFasL were significantly increased in SS patients [24], which is contrary to our results here. The difference might be resulting from the various race, age, treatment, and disease duration time of subjects. In our study, sFasL was found decreased in SS patients and the sFasL levels were positively correlated with uptake index of parotid gland in the SS patients, and the SS patients with PBS/AIH showed a decreased expression of sFasL in sera. These data showed that sFasL may prevent damage of gland injury from active lymphocytes through inducing apoptosis.

Imbalance of cytokines also was implicated in the damage of gland in SS patients. It has been suggested that $\mathrm{T}$ helper type 1 (Th1) cytokines, such as IFN- $\gamma$, IL-2, and IL-6, might be important in the induction and/or maintenance of SS [38]. Recently, activated $\mathrm{T}$ cells remain resistant to Fas stimulation and their sensitivity to apoptosis increases gradually, regulated by a number of interrelated factors, including availability of FasL-presenting cells and the pro- and antiapoptotic factors and the presence of cytokines [11, 39-41]. Previous study demonstrated that the Breg cells and Th1, Th17, and Th2 2 cells showed a negative correlation, and IL-10, IFN- $\gamma$, IL-17, and IL-22 levels expressed by these cells also showed a negative correlation [42]. These activities are mediated, at least in part, by the production of IL10 and may control a variety of autoinflammatory diseases including inflammatory arthritis, inflammatory bowel disease, autoimmune diabetes, SLE, and SS [43]. Inflammatory cytokines (IL-2, IL-5, IL-6, and IL-12) tend to protect Th1 cells from apoptosis, while immunomodulatory/anti-inflammatory ones like IL-10 have a proapoptotic effect $[31,44-46]$. In our study, a positive correlation of sFasL with IL-10 was observed. sFasL can promote apoptosis of immune cell resulting in preventing parotid gland injury in Sjogren's syndrome. At the same time, we also found that sFasL might have a protective role in the damage of liver in SS patients with AIH.

In conclusion, the results presented here suggest that the serum sFasL may play a beneficial role in the pathogenesis of SS gland injury. These data maybe suggest a novel approach in the SS treatment. Certainly, further studies are required to explore the specific regulation mechanism between sFasL and SS.

\section{Competing Interests}

The authors declare no conflict of interests regarding the publication of this paper.

\section{Authors' Contributions}

Jiao Luo and Ying Wang contributed equally to this work. 


\section{Acknowledgments}

This work was supported by the National Natural Science Foundation of China (NSFC) U1605223 to Guixiu Shi and 81671544 to Hongyan Qian and Major disease research projects of Xiamen no. 3502Z20149029 to Guixiu Shi.

\section{References}

[1] F. N. Skopouli, U. Dafni, J. P. A. Ioannidis, and H. M. Moutsopoulos, "Clinical evolution, and morbidity and mortality of primary Sjogren's syndrome," Seminars in Arthritis and Rheumatism, vol. 29, no. 5, pp. 296-304, 2000.

[2] A. S. Malladi, K. E. Sack, S. C. Shiboski et al., "Primary Sjögren's syndrome as a systemic disease: a study of participants enrolled in an International Sjögren's syndrome registry," Arthritis Care \& Research, vol. 64, no. 6, pp. 911-918, 2012.

[3] C. P. Mavragani and H. M. Moutsopoulos, "The geoepidemiology of Sjögren's syndrome," Autoimmunity Reviews, vol. 9, no. 5, pp. A305-A310, 2010.

[4] S. Lindgren, R. Manthorpe, and S. Eriksson, "Autoimmune liver disease in patients with primary Sjögren's syndrome," Journal of Hepatology, vol. 20, no. 3, pp. 354-358, 1994.

[5] G. S. Hatzis, G. E. Fragoulis, A. Karatzaferis, I. Delladetsima, C. Barbatis, and H. M. Moutsopoulos, "Prevalence and longterm course of primary biliary cirrhosis in primary Sjögren's syndrome," Journal of Rheumatology, vol. 35, no. 10, pp. 2012-2016, 2008.

[6] M. Ramos-Casals, J.-M. Sánchez-Tapias, A. Parés et al., "Characterization and differentiation of autoimmune versus viral liver involvement in patients with Sjögren's syndrome," Journal of Rheumatology, vol. 33, no. 8, pp. 1593-1599, 2006.

[7] S. Yonehara, A. Ishii, and M. Yonehara, "A cell-killing monoclonal antibody (anti-Fas) to a cell surface antigen codownregulated with the receptor of tumor necrosis factor," Journal of Experimental Medicine, vol. 169, no. 5, pp. 1747-1756, 1989.

[8] T. Takahashi, M. Tanaka, J. Inazawa, T. Abe, T. Suda, and S. Nagata, "Human fas ligand: gene structure, chromosomal location and species specificity," International Immunology, vol. 6, no. 10, pp. 1567-1574, 1994.

[9] S. Nagata and P. Golstein, "The fas death factor," Science, vol. 267, no. 5203, pp. 1449-1456, 1995.

[10] P. H. Krammer, J. Dhein, H. Walczak et al., "The role of APO1-mediated apoptosis in the immune system," Immunological Reviews, vol. 142, pp. 175-191, 1994.

[11] M. R. Alderson, T. W. Tough, T. Davis-Smith et al., "Fas ligand mediates activation-induced cell death in human T lymphocytes," Journal of Experimental Medicine, vol. 181, no. 1, pp. 71-77, 1995.

[12] D. Bellgrau, D. Gold, H. Selawry, J. Moore, A. Franzusoff, and R. C. Duke, "A role for CD95 ligand in preventing graft rejection," Nature, vol. 377, no. 6550, pp. 630-632, 1995.

[13] T. S. Griffith, T. Brunner, S. M. Fletcher, D. R. Green, and T. A. Ferguson, "Fas ligand-induced apoptosis as a mechanism of immune privilege," Science, vol. 270, no. 5239, pp. 1189-1192, 1995.

[14] J. S. Hunt, D. Vassmer, T. A. Ferguson, and L. Miller, "Fas ligand is positioned in mouse uterus and placenta to prevent trafficking of activated leukocytes between the mother and the conceptus," Journal of Immunology, vol. 158, no. 9, pp. 4122-4128, 1997.
[15] P. G. Knox, A. E. Milner, N. K. Green, A. G. Eliopoulos, and L. S. Young, "Inhibition of metalloproteinase cleavage enhances the cytotoxicity of Fas ligand," The Journal of Immunology, vol. 170, no. 2, pp. 677-685, 2003.

[16] N. Li, H. Nie, Q.-W. Yu et al., "Role of soluble Fas ligand in autoimmune diseases," World Journal of Gastroenterology, vol. 10, no. 21, pp. 3151-3156, 2004.

[17] R.-P. Jia and X.-Y. Zhao, "[Expression and clinical significance of CD4(+)CD25(+)Treg cells, sFas and sFasL in peripheral blood of patients with autoimmune thrombocytopenic purpura]," Zhongguo shi yan xue ye xue za zhi, vol. 19, no. 5, pp. 1264-1267, 2011.

[18] C. Vitali, S. Bombardieri, R. Jonsson et al., "Classification criteria for Sjögren's syndrome: a revised version of the European criteria proposed by the American-European Consensus Group," Annals of the Rheumatic Diseases, vol. 61, no. 6, pp. 554$558,2002$.

[19] C. Vitali, G. Palombi, C. Baldini et al., “Sjögren's syndrome disease damage index and disease activity index: scoring systems for the assessment of disease damage and disease activity in Sjögren's syndrome, derived from an analysis of a cohort of Italian patients," Arthritis and Rheumatism, vol. 56, no. 7, pp. 2223-2231, 2007.

[20] G. Hernández-Molina and T. Sánchez-Hernández, "Clinimetric methods in Sjögren's syndrome," Seminars in Arthritis and Rheumatism, vol. 42, no. 6, pp. 627-639, 2013.

[21] R. Seror, J. E. Gottenberg, V. Devauchelle-Pensec et al., "European league against Rheumatism Sjögren's syndrome disease activity index and European League Against Rheumatism Sjögren's syndrome patient-reported index: a complete picture of primary Sjögren's syndrome patients," Arthritis Care and Research, vol. 65, no. 8, pp. 1358-1364, 2013.

[22] E. H. Kang, Y. J. Lee, J. Y. Hyon, P. Y. Yun, and Y. W. Song, "Salivary cytokine profiles in primary Sjögren's syndrome differ from those in non-Sjögren sicca in terms of TNF- $\alpha$ levels and Th-1/Th-2 ratios," Clinical and Experimental Rheumatology, vol. 29, no. 6, pp. 970-976, 2011.

[23] M. D. Mignogna, S. Fedele, L. Lo Russo, L. Lo Muzio, and A. Wolff, "Sjögren's syndrome: the diagnostic potential of early oral manifestations preceding hyposalivation/xerostomia," Journal of Oral Pathology and Medicine, vol. 34, no. 1, pp. 1-6, 2005.

[24] K. Nozawa, N. Kayagaki, Y. Tokano, H. Yagita, K. Okumura, and H. Hasimoto, "Soluble Fas (APO-1, CD95) and soluble Fas ligand in rheumatic diseases," Arthritis and Rheumatism, vol. 40, no. 6, pp. 1126-1129, 1997.

[25] K. Musiał and D. Zwolińska, “The sFas/sFasL ratio as a novel marker of inflammation in children with chronic kidney disease," Clinica Chimica Acta, vol. 414, pp. 7-11, 2012.

[26] J. Haddad, J.-C. Trinchet, D. Pateron et al., "Lymphocytic sialadenitis of Sjögren's syndrome associated with chronic hepatitis C virus liver disease," The Lancet, vol. 339, no. 8789, pp. 321-323, 1992.

[27] G. Xanthou, N. I. Tapinos, M. Polihronis, I. P. Nezis, L. H. Margaritis, and H. M. Moutsopoulos, "CD4 cytotoxic and dendritic cells in the immunopathologic lesion of Sjogren's syndrome," Clinical and Experimental Immunology, vol. 118, no. 1, pp. 154-163, 1999.

[28] C. Bodeutsch, P. C. M. De Wilde, L. Kater et al., "Monotypic plasma cells in labial salivary glands of patients with Sjogren's syndrome: prognosticator for systemic lymphoproliferative disease," Journal of Clinical Pathology, vol. 46, no. 2, pp. 123-128, 1993. 
[29] D. I. Stott, F. Hiepe, M. Hummel, G. Steinhauser, and C. Berek, "Antigen-driven clonal proliferation of B cells within the target tissue of an autoimmune disease. The salivary glands of patients with Sjogren's syndrome," Journal of Clinical Investigation, vol. 102, no. 5, pp. 938-946, 1998.

[30] T. Dörner and P. E. Lipsky, "Abnormalities of B cell phenotype, immunoglobulin gene expression and the emergence of autoimmunity in Sjögren's syndrome," Arthritis Research, vol. 4, no. 6, pp. 360-371, 2002.

[31] A. N. Akbar, N. Borthwick, M. Salmon et al., "The significance of low bcl-2 expression by CD45RO T cells in normal individuals and patients with acute viral infections. The role of apoptosis in T cell memory," Journal of Experimental Medicine, vol. 178, no. 2, pp. 427-438, 1993.

[32] G. Scaffidi, I. Schmitz, J. Zha, S. J. Korsmeyer, P. H. Krammer, and M. E. Peter, "Differential modulation of apoptosis sensitivity in CD95 type I and type II cells," Journal of Biological Chemistry, vol. 274, no. 32, pp. 22532-22538, 1999.

[33] A. K. Vaishnaw, J. D. McNally, and K. B. Elkon, "Apoptosis in the rheumatic diseases," Arthritis and Rheumatism, vol. 40, no. 11, pp. 1917-1927, 1997.

[34] N. Ogawa, H. Dang, L. Kong, J.-M. Anaya, G. T. Liu, and N. Talal, "Lymphocyte apoptosis and apoptosis-associated gene expression in sjogren's syndrome," Arthritis and Rheumatism, vol. 39, no. 11, pp. 1875-1885, 1996.

[35] M. Rogge, X.-T. Yin, L. Godfrey et al., "Therapeutic use of soluble fas ligand ameliorates acute and recurrent herpetic stromal keratitis in mice," Investigative Ophthalmology and Visual Science, vol. 56, no. 11, pp. 6377-6386, 2015.

[36] J. Perosso, K. L. O. Silva, S. Í. D. S. Ferreira et al., "Alteration of sFAS and sFAS ligand expression during canine visceral leishmaniosis," Veterinary Parasitology, vol. 205, no. 3-4, pp. 417-423, 2014.

[37] Y. Qin, P. Liao, S. He et al., "Detection of FasL mRNA, sFasL and their regulatory effect on T lymphocyte subsets in patients with severe acute pancreatitis," Chinese Journal of Cellular and Molecular Immunology, vol. 29, no. 11, pp. 1189-1192, 2013.

[38] N. Amft and S. J. Bowman, "Chemokines and cell trafficking in Sjögren's syndrome," Scandinavian Journal of Immunology, vol. 54, no. 1-2, pp. 62-69, 2001.

[39] M. Li-Weber and P. H. Krammer, "Function and regulation of the CD95 (APO-1/Fas) ligand in the immune system," Seminars in Immunology, vol. 15, no. 3, pp. 145-157, 2003.

[40] T. Miyawaki, T. Uehara, R. Nibu et al., "Differential expression of apoptosis-related fas antigen on lymphocyte subpopulations in human peripheral blood," Journal of Immunology, vol. 149, no. 11, pp. 3753-3758, 1992.

[41] I. Schmitz, A. Krueger, S. Baumann, H. Schulze-Bergkamen, P. H. Krammer, and S. Kirchhoff, "An IL-2-dependent switch between CD95 signaling pathways sensitizes primary human T cells toward CD95-mediated activation-induced cell death," Journal of Immunology, vol. 171, no. 6, pp. 2930-2936, 2003.

[42] C.-L. Wu, Q. Wang, L. Zheng, D.-Y. Gu, J.-A. He, and C.-P. Shao, "[Correlation of Breg with CD4(+)T cells of peripheral blood in patients with CITP and its clinical significance]," Journal of experimental hematology/Chinese Association of Pathophysiology, vol. 21, no. 6, pp. 1517-1521, 2013.

[43] K. Yanaba, J.-D. Bouaziz, K. M. Haas, J. C. Poe, M. Fujimoto, and T. F. Tedder, "A regulatory B cell subset with a unique CD1dhiCD5+ phenotype controls T cell-dependent inflammatory responses," Immunity, vol. 28, no. 5, pp. 639-650, 2008.
[44] T. Uehara, T. Miyawaki, K. Ohta et al., "Apoptotic cell death of primed CD45RO+ $\mathrm{T}$ lymphocytes in Epstein-Barr virusinduced infectious mononucleosis," Blood, vol. 80, no. 2, pp. 452-458, 1992.

[45] J. Estaquier, T. Idziorek, W. Zou et al., “T helper type 1/T helper type 2 cytokines and $T$ cell death: preventive effect of interleukin 12 on activation-induced and CD95 (FAS/APO-1)-mediated apoptosis of CD4+ T cells from human immunodeficiency virus-infected persons," Journal of Experimental Medicine, vol. 182, no. 6, pp. 1759-1767, 1995.

[46] G. Roth, B. Moser, C. Krenn et al., "Susceptibility to programmed cell death in T-lymphocytes from septic patients: a mechanism for lymphopenia and Th2 predominance," Biochemical and Biophysical Research Communications, vol. 308, no. 4, pp. 840-846, 2003. 


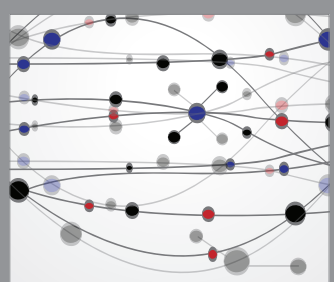

The Scientific World Journal
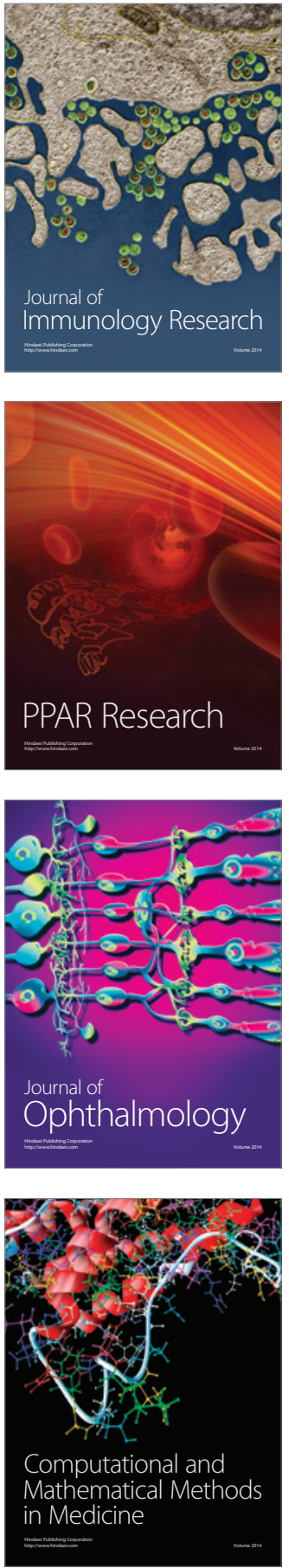

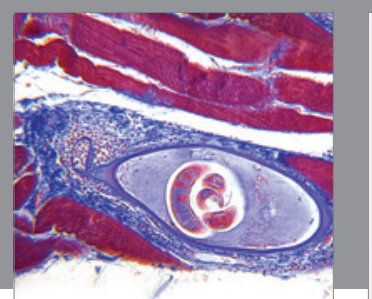

Gastroenterology Research and Practice
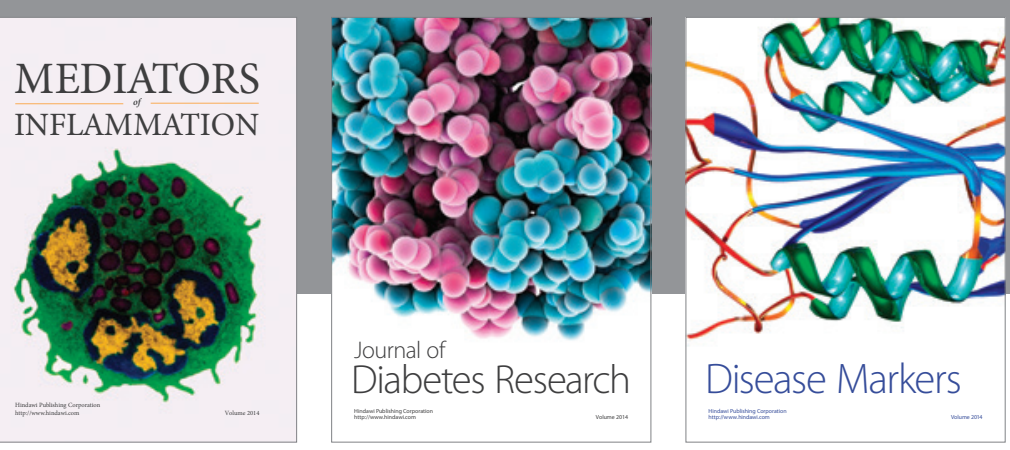

Disease Markers

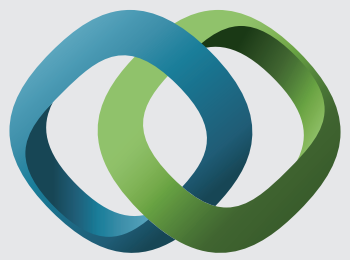

\section{Hindawi}

Submit your manuscripts at

https://www.hindawi.com
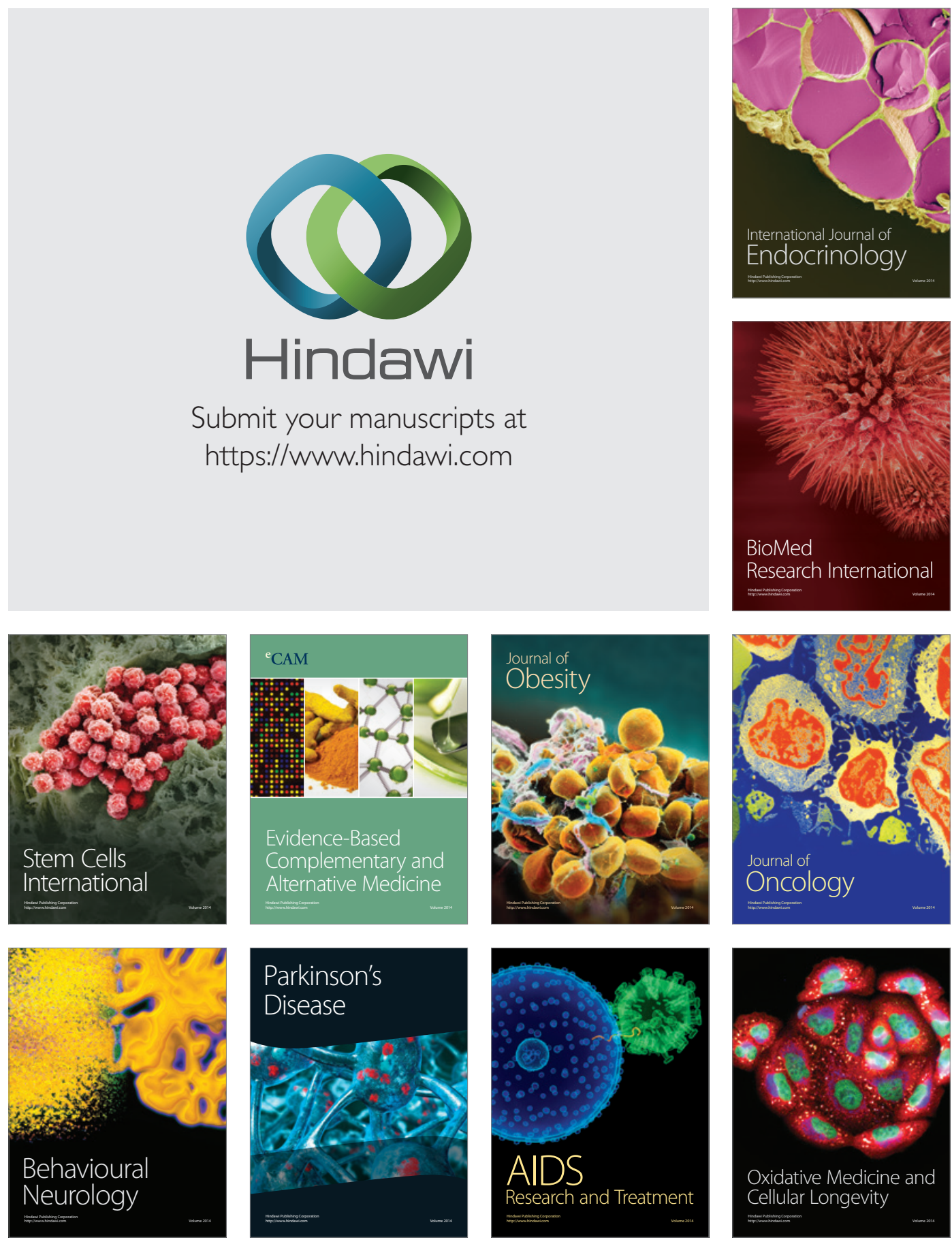